\title{
Naturalistic Intelligence and Environmental Awareness among Graduate Students
}

\author{
Zarah Beby Ningrum ${ }^{1, *}$, Tri Edhi Budhi Soesilo ${ }^{1}$, and Herdis Herdiansyah ${ }^{1}$ \\ ${ }^{1}$ School of Environmental Science, Universitas Indonesia, 10430, Salemba, Indonesia
}

\begin{abstract}
Students in universities being an important part of responses to reduce environmental problems and bear profound responsibilities to increase the awareness to create on environmentally sustainable future. The study focuses on the naturalistic intelligences from one of Multiple Intelligences Theory by Howard Gardner. This study aimed to analyze the relationship between naturalistic intelligence with environmental awareness on graduate students at University of Indonesia. In this study, naturalistic intelligence is the independent variable while environmental awareness is the dependent variable. The students who were selected randomly will be given a questionnaire as a research instrument. Afterward, a correlation analysis was conducted with Spearmann test. This study shows that environmental science students had high naturalistic intelligence and good environmental awareness. The analysis shows that there is a strength and significant correlation between the naturalistic intelligence with environmental awareness among graduate students with the value of coefision correlation is 0,754 . The students who interest in flora and fauna, understanding the environmental problems, enjoy outdoor activities, have scientific hobbies and concern to the change of environmental will also have a high awareness of the environment. The naturalistic intelligence of the students possess also develops a positive attitude toward the environment and directs the students to have a proenvironmental behavior.
\end{abstract}

\section{Introduction}

Human activities have been harmful and cause changes to the environment. This is indicated by the activity that produces chemicals to the soil, air, and water in the industry in Indonesia. This is evidenced by the monitoring results of the Ministry of Environment and Forestry in 2016. The presence of garbage and household waste accumulation also causes pollution and environmental damage. Many studies have shown that environmental damage caused by human activity [1]. Nevertheless, humans are the main managers of the environment with the ability and expertise to manage the environment and utilize the existing resources on an ongoing basis.

Students as one of the elements in society is expected to be the next generation and the developer to preserve the environment and resources available. Education obtained by students at an institution will have a positive impact in improving the ability and environmental awareness of students who are studying specifically about the environment 
and its problems. Environmental education for young people is the most important, as young people are the main driver of a more sustainable future, especially students who occupy higher education [2]. Students in higher education or universities being an important part of responses to reduce environmental problems and bear profound responsibilities to increase the awareness to create on environmentally sustainable future

However, Kumurur's study indicates that students' environmental awareness is still low. There are still students who rarely engage in environmentally friendly activities such as doing electricity savings or utilizing and maintaining the cleanliness of public transportation [3]. In addition, Oguz et al., found that only half of all students do recycling [4]. Environmental awareness among students is important to be implemented from the education gained by preserving the environment and the resources existing on earth [5]. The less maximal behavior and environmental awareness on students are likely to occur because of the differences in students' cognitive abilities. This is explained by Gardner as a difference in human intelligence known as multiple intelligence [6].

Naturalistic intelligence is a new addition that meets the criteria as an intelligence. It is a skill that a person possesses in recognizing and classifying various species (flora and fauna) found in the environment. This capability also includes sensitivity to natural phenomena [7]. A person who has naturalistic intelligence and continues to develop it will be able to maintain the environment and know the consequences of his actions to nature $[7,8]$.

Therefore, it is necessary to ensure that the naturalistic intelligence contributes to the positive attitudes and behaviors towards the environment. Further, analysis needs to be carried out about the relationship between naturalistic intelligence and environmental awareness among students in dealing with environmental problems. Since there have yet to be any literature studies regarding this topic, this study needs to be done in an effort to improve students' environmental awareness. This research can contribute to the optimization of campus management and learning systems about the environment for environmental sustainability by utilizing the naturalist intelligence of its students college.

\subsection{Naturalistic Intelligence}

Intelligence is a bio-psychological potential for processing information that can be activated in a cultural environment to solve problems or create cultural valued products [6]. Gardner developed a theory called Multiple Intelligence which was divided into seven types of intelligence, namely linguistic intelligence, logical-mathematical intelligence, visual-spatial intelligence, bodily kinesthetic intelligence, musical intelligence, interpersonal intelligence, interpersonal intelligence. While in 1999, Gardner added two new categories which are naturalistic intelligence and extensional intelligence [8].

This study focuses on naturalistic intelligence on students of environmental science. Naturalistic intelligence is selected because it becomes one of the dominant intelligences in a person who focuses on understanding the environment. A person who understands nature should have a developed naturalistic intelligence because they are in a place surrounded by natural experts and often engaged in direct interaction with them. It becomes a factor that will increase their knowledge of nature and develop their naturalist intelligence.

Naturalistic intelligence is the ability to understand the natural environment well, can create other consequential distinctions in the nature of nature; the ability to understand and enjoy nature, and use that ability productively [8]. Naturalistic intelligence is defined as the expertise in identifying and classifying various species (flora and fauna) that exist in the individual's environment, it also includes sensitivity to other natural phenomena (e.g., cloud formations, mountains, and others) [7]. Naturalistic intelligence can be measured by characteristics [7] as follows: 
1. Having sensory senses that are sensitive to environmental changes.

2. Being able to identify or classify living things and other things that exist in nature.

3. Appreciate being outside and enjoying outdoor activities.

4. Being interested and caring for the living things and the environment.

5. Frequently identifying something in the environment.

6. Creating, keeping or having their own collections, journals, natural objects, images, photographs and specimens.

7. Being very interested in lessons or television shows, videos, books or objects about nature.

8. Showing awareness of the environment and its changes.

9. Finding it easy to learn characteristics, names, categorization, and data about objects or species found in nature.

10. Concern to things that others may not realize in nature.

The indicator selected from the characters for this study are interet flora and fauna (living things), understanding the environmental problems, outdoor activities, scientific hobbies, and environmental concern. Naturalistic intelligence can be identified through environmental awareness, such as following every activity related to environmental conservation [9]. Someone with naturalistic intelligence will commit to not cutting trees indiscriminately, destroying the surrounding plants, torturing animals, hunting animals. Instead, they will keep the surrounding environment clean and sustainable. This naturalistic intelligence is dominantly possessed by biologists, nature lovers, environmental activists, etc. Hayes said students who are invited to involve in direct observation to nature will have the knowledge of recognizing the nature well and its potential more deeply [10].

\subsection{Environmental Awareness}

Environmental awareness is defined as the emotional level and commitment to environmental issues [11]. It also means a person's attitude to pay special attention in maintaining and respecting the environment [12]. It can also be interpreted as attitudes toward the environment [13]. Furthermore, environmental awareness can be identified by the amount of empirical knowledge, emotional degree, level of desire, and the extent of actual behavior on environmental issues ${ }^{[14]}[12][15]$.

In the previous studies of environmental issues, the measurement was done by using the basic factor of attitudes because environmental awareness have been interpreted as a form of positive attitude to the environment. It also called pedagogical components and it has three main dimensions: cognitive, affective, and behavior [16]. The cognitive component, which refers to the mental processes of perception, conception and beliefs about attitudes object. Affective or emotional components, which collect all emotions and feelings, for example; subjective reactions of trust and distrust, likes and dislikes, among others. Finally, conative or behavioral components are related to expressions of behavioral intention or action, behaviors that represent a tendency to act or resolve problem in a spesific way. Therefore, environmental awareness can also be interpreted with a tendency to behave in a pro-environment manner [17]. These three components will be the scale for measuring environmental awareness in this study.

In fact, many scales have been used as an environmental awareness scale, in comparison there are three widely used scales [18] such as Ecological Scale [16], Environmental Awareness Scale [19], and New Environmental Paradigm Scale [20,21]. High 
environmental awareness might be an important prerequisite of pro-environment longlasting behavior, thereby reducing environmental impacts continuously [13].

\section{Materials and Methods}

The goal of this research is to analyze the relationship of naturalistic intelligence with environmental awareness among graduate students. This study is conducted with quantitative and qualitative method. Quantitative data obtained from research instrument in the form of questionnaire and qualitative data obtained from interview selected students.

The research is located in Central Jakarta, as one of the municipalities that becomes the center of community activity in Jakarta. University of Indonesia was chosen because it is one of the institutions located in the city center and has a special department studying environmental science. The population of this study is all active postgraduate students in the School of Environmental Science, University of Indonesia, which is amounted to 216 students. The number of sample corresponds to a population definition whose minimum number is set by using the Slovin formula:

$$
n=\frac{N}{1-N \alpha^{2}}
$$

Information:

$\mathrm{n} \quad=$ Number of samples

$\mathrm{N}=$ Number of population

$\alpha=$ degree of error set by the researcher of $10 \%$

Based on the calculations, 68 graduate students were selected as the respondents of this study, 30 students are female and 38 students are male. For the interview, 15 people were selected. Before performing the analysis of realtionship, data were collected for the validation and reliability of the questionnaire to be used. The questionnaires that have been proven valid and reliable were then used as a variable measurement tool.

The questionnaire has three parts. Part one provides backgrounds on respondents regarding age, gender, recent education, and residence. The second part measures the naturalistic intelligence, while the third part measures the students' environmental awareness of environmental issues in a variety of ways, such as responding to environmental problems, energy-saving attitudes, and responsibility for protecting the environment.

Some items for the survey were mainly derived from numerous studies in previous studies. Meanwhile, other statements were designed in accordance with the actual situation such as the current conditions. The validity and reliability test were firstly carried out to instruments that will be used as a measuring tool. The validity test uses Product Moment and the reliability test uses Cronbach Alpha. Instruments created by using a Likert scale has four alternative answers; Strongly, Agree, Disagree, and Strongly Disagree.

Furthermore, the data analysis was done with SPSS program version 22.00. The analysis applied the bivariate correlation (Spearman correlation test). This analysis was done to obtain the value of correlation coefficient of two variables - independent variables and dependent variables - which were allegedly related or correlated. The correlation coefficient is obtained to see the size and strength of the relationship between naturalistic intelligence and environmental awareness. In addition, correlation test will also allow the determination of the correlation relationship criteria. 


\section{Results and Discussions}

\subsection{Validity and Reliability}

The research began with performing validity and reliability tests on instruments used to measure naturalistic intelligence and environmental awareness among students. The 40 items of naturalistic intelligence statements, 28 items of valid statements are identified. Reliability test indicated that alpha $0.892>0.444$ which means the items on the questionnaire are reliable as a means of collecting data in this study. While 20 items of a valid statement are identified from 24 items of environmental awareness statement. Reliability test showed alpha that $0.881>0.444$ which means the items on the questionnaire are reliable as a means of collecting data in this study.

\subsection{The Relationship Between Naturalistic Intelligence and Environmental Awareness}

The results show that environmental science students had high naturalistic intelligence because it has an average value of 89.51 . The lowest value of the naturalistic intelligence is 80 which is obtained by two students and the highest value of 104 is obtained by one student. As for the environmental awareness, the average value is 65.16 with the lowest value of 57 which is obtained by three students and the highest value of 77 is obtained by one student. It shows that the environmental awareness of the students is average because 32 students has medium value.

Spearman test is used to observe the relationship between two variables. This test explains the strength of the relationship and whether there is a significant or no relationship between the naturalistic intelligence and students' environmental awareness. The significant level used is the confidence level of P 0.05 . The relationship is investigated between the naturalistic intelligence and environmental awareness.

Table 1. Correlation Coefficient Value

\begin{tabular}{|c|c|c|}
\hline $\begin{array}{c}\text { Variable X } \\
\text { and Y }\end{array}$ & $\begin{array}{c}\text { Correlation } \\
\text { "r" }\end{array}$ & p-value \\
\hline $\begin{array}{c}\text { Naturalistic } \\
\text { Intelligence and } \\
\text { Environmental } \\
\text { Awareness }\end{array}$ & 0,754 & 0.000 \\
\hline
\end{tabular}

The results show that there is positive correlation between the naturalistic intelligence with environmental awareness among graduate students. The correlation coefficient value of the results is 0.754 which means there is a strength and significant relationship between the two variables. Where the higher value of naturalist intelligence, the higher value of the environmental awareness. Applicable otherwise the lower the value of the attitude of preserving the environment will be lower also the value of behavior to preserve the environment.

Statistical analysis shows that there is a low and significant relationship between interest in flora and fauna with students' cognitive in paying attention at the environment 
and its problems. Further, there is a moderate relationship found between students' understanding of environmental problems and outdoor activities with students' cognitive. While between the scientific hobbies and environmental awareness, there is no significant relationship found. This suggests that increasing interest in flora and fauna, environmental problems understanding and outdoor activities will also increase the students' knowledge, views and opinions to deal with environmental problems.

Statistical analysis also shows that all components of naturalistic intelligence with affective component of environmental awareness indicates a significant relationship between them. Furthermore, the correlation is found in the overall components of naturalistic intelligence with one of the last components of environmental awareness which is behavior. But the correlation is weak, this means that any increase in interest in flora and fauna, understanding the environmental problems, outdoor activities, scientific hobbies, and awareness of environmental changes will also make students behave positively toward the environment. Positive behavior that students tend to show based on interviews is to save electricity by turning off lights when leaving the room, following organizations that play a role in the environment, and using recyclable containers when shopping.

Naturalist intelligence will ultimately produce students who are pro-environmentally, and have an attitude towards the environment. Structurally will also make campus management more environmentally friendly [22][23] and these conditions will apply in other education areas. Meanwhile, there are other factors that have a relationship and influence, positive and negative on environmental awareness such as knowladge, environmental education, socio-demographic factors [24], gender [25], the educational status of the parents of the students, their living area in which they lived most of the time, their status of having hobbies [26], and culture (the communities which still have strong customary law will tend to preserve the environment and limit usages that cause damage to the environment) [27].

\section{Conclusion}

This study concludes that environmental science students had high naturalistic intelligence and good environmental awareness. There is a strength and significant correlation between the naturalistic intelligence with environmental awareness among graduate students. Besides, students' naturalistic intelligence develops the environmental awareness or positive attitudes toward the environment and directs students to have proenvironmental behavior. Meanwhile, there are other factors that have a relationship and influence, positive and negative on environmental awareness such as knowladge, environmental education, socio-demographic factors, the educational status of the parents of the students, their living area in which they lived most of the time, their status of having hobbies, and culture.

\section{Acknowledgments}

This research is funded by the Grant of Indexed International Publication for Final Project of Students Publikasi Terindeks Internasional untuk Tugas Akhir Mahasiswa (PITTA) Universitas Indonesia 2018 with contract number 2577/UN.R3.1/HKP.05.00/2018.

\section{References}

1. Koger, S. Sustainability 5, 7 (2013). 
2. Green, T. L. Ecol. Econ 94 (2013).

3. Kumurur,S. et al. J. Ekoton 8, 2 (2008).

4. Oguz, D.,I. Çakci, and S. Kavas.African. J. Agric. Res 5, 19 (2010).

5. Sivamoorthy, M., R. Nalini, and C. S. Kumar. Int. J. Humanit. Soc. Sci. Invent 2, 8 (2013).

6. Gardner, H.. Frames of mind, (Basic Books, New York, 1988).

7. Armstrong, T. and B. Franklin, Multiple intelligences in the classroom, (ASCD, USA, 2009)

8. Gardner, H.. Intelligence reframed: multiple intelligences, (Basic Books, New York, 1999)

9. Lazear, D. G. Development of multiple intelligences. (McMillan Inc, New York, 2000)

10. Hayes, M. A. Conserv. Biol 23, 5 (2009).

11. Aman, A. H. L., A. Harun, Z. Hussein, and C. Author.Br. J. Arts Soc. Sci 7, 2 (2012).

12. Fransson,N. and T. Gärling. J. Environ. Psychol 19, 4 (1999).

13. Takács-Sánta,A. Hum. Ecol. Rev 14, 1 (2007).

14. Maloney, M. P., M. P. Ward, and G. N. Braucht 30, 7 (1975).

15. Ugulu, I., M. Sahin, and S. Baslar. Int. J. Educ. Sci 5, 4 (2013).

16. Garcia-Santillan, A., E. Moreno-Garcia, J. Carlos-Castro, J. H. Zamudio-Abdala, and J. Garduno-Trejo. J. Math. Res 4, 5 (2012).

17. Stern,P. C.J. Soc. Issues 56, 3 (2000).

18. Dunlap, R. E. and R. E. Jones. Environmental concern: conceptual and measurement issues, (Greenwood Press., Westport, CT, 2002).

19. Weigel, R. and J. Weigel. Environ. Behav 10, 1 (1978).

20. Dunlap,R. E. and K. D. Van Liere. J. Environ. Educ 9, 4 (1978).

21. Dunlap,R. E. J. Soc. Iss 56, 9 (2000)

22. Herdiansyah, H. T. Jokopitoyo, and A. Munir. IOP Conf. Ser. Ser.: Earth Environ. Sci (2016).

23. Herdis Herdiansyah et al. IOP Conf. Ser.: Earth Environ. Sci (2017).

24. Bozoglu,M., A. Bilgic, B. K. Topuz, and Y. Ardali.Fresenius Environ. Bull. 25, 4 (2016)

25. Nafi, T. H. R. Lestarini, and S. Wulandhary. E3S Web of Conferences 52 (2018)

26. Tekin, C. and G. Gunes. Int J Community Med Public Health 5, 2 (2018).

27. Lestarini,R., R. Harmain, S. Wulandhary, and D. Utari. E3S Web of Conferences 52 (2018). 